\title{
Carbon Nanotube Structures: Y-Junctions and Nanorings
}

\author{
A. Latgé, R. B. Muniz, and D. Grimm \\ Instituto de Física, Universidade Federal Fluminense \\ Av. Litorânea sn, 24210-340 Niterói, Rio de Janeiro, Brazil
}

Received on 31 March, 2003

\begin{abstract}
The possibility of coupling two Y-junctions of carbon nanotubes, forming a type of nanotube ring is addressed here. Recent experimental evidences of growing carbon nanotube structures forming basically Y-kind junctions give support to this theoretical proposition. A single-band tight-binding Hamiltonian is considered and local electronic properties are investigated via the Green function formalism following renormalization techniques. Modulation on the transport properties of those nanostructured systems may be induced by considering different kind of junctions and different symmetries. Quantum interference phenomena are proven to reduce partial or completely the conductance of such nanoring structures.
\end{abstract}

\section{Introduction}

Single-wall carbon nanotube junctions have recently appeared as excellent candidates for nanoscale multi-terminal electronic devices. Experimental reports have presented clear support on the possibility of synthesizing stable junctions incorporating mainly two quite different approaches. Controlled growth using a template-based chemical vapor deposition technique [1] allows reproducible and high-yield fabrication of Y-junctions. Another procedure allows welding together two crossed carbon-nanotubes under quite high temperatures (around $800^{\circ} \mathrm{C}$ ), by exposing to a controlled electron beam $[2,3]$. Continuous sputtering of carbon atoms from the perfect nanotubes lead to dimensional changes and surface reconstruction with annealing at their contact region forming a X-like junction with diverse angles between the branches. Under careful irradiation one of the branches of the X-junction can be removed, creating Y- and T-like junction [2], denoted in what follows by YJ. Experimental [4] and theoretical [5] studies have shown, that this particular new class of carbon junctions exhibits an intrinsic non-linear transport behavior, depending mainly on pure geometrical configuration and on the kind of the topological defects used to build the YJ. Calculations and measurement of characteristic curves like current vs. voltage of different sets of YJ show robust rectification properties giving rise to the possibility of using YJ as a nanoscale three-point transistor.

Alternatively, carbon-nanotube ring transistors have been proposed experimentally [6]. Under ultrasonic irradiation, pure nanotubes can fold to form nanotube rings with controlled ring diameter. To measure the currentvoltage characteristic of these rings a scanning tunneling microscope was used with two pure carbon nanotubes as dual probe allowing an image-resolution of about $1 \mathrm{~nm}$. Theoretical studies show, again, that mainly the topology and structure details determine the electronic properties of the ring [7].

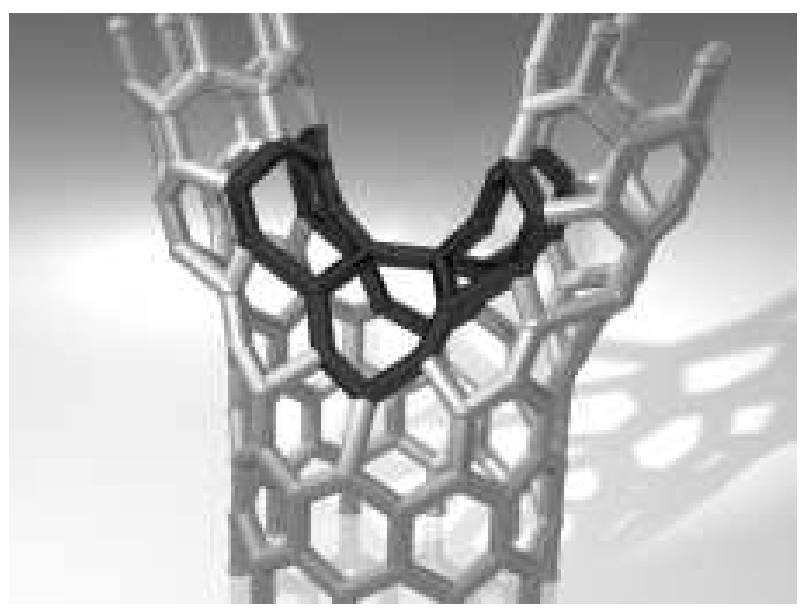

Figure 1. The 6 heptagon topological defect of the Y-junction.

By taking into account both carbon nanotube (CNT) configurations, YJs and rings, here we propose a new class of nanoscale device similar to the above mentioned ring transistor configuration. Joining the smaller arms of two YJs, a nanotube ring system may be idealized with two attached nanotube leads, called here as an Y-junction ring (YJR). The electronic characteristics of the carbon nanotube together with the annular geometry of those rings can be very useful for investigating interesting physical phenomena such as Aharonov-Bohm effects [8]. Here we investigate the dependence of constructive or destructive quantum phenomena in function of the branch lengths and diameters. In all studied symmetry geometries the conductance exhibit, near the Fermi-energy, a reduction of the quantum channels when compared to the case of pure CNT. We also show the transistor-like behavior controlled by one finite arm of the YJ and compare it to the pure one-dimensional case and a 5-7 CNT-heterojunction previously studied[9]. 


\section{Y-Junction}

A pure CNT can be uniquely determined by the chiral vector $C=n a_{1}+m a_{2}$, where $n$ and $m$ are integers and $a_{1}$ and $a_{2}$ are the graphene sheet lattice vectors, with $\left|a_{i}\right| \approx 2,49$ $\AA$. The $(n, m)$ CNTs are metallic, if $n-m$ is a multiple of three and semiconducting otherwise, i.e., the armchair CNTs $(n, n)$ are always metallic whereas the zigzag CNTs $(n, 0)$ requires an $n$ multiple of 3 .

The Y-junction is modeled here joining three arms of zigzag CNTs together maintaining the original chirality. The large zigzag $(\mathrm{m}, 0) \mathrm{CNT}$ bifurcates into two smaller zigzag CNTs, $(\mathrm{n}, 0)$ and $(\tilde{n}, 0)$, forming an acute angle between the smaller arms, exactly where six heptagons are supposed to be fixed as a topological defect. This YJ is denoted as $m \rightarrow n / \tilde{n}$. Fig. 1 illustrates the chosen YJ described here. The defect atoms, forming six heptagons according to the Crespi rule[10], are marked with dark lines. Independently of the tube diameters composing the YJ, the number of atoms in each cross section, before and after the bifurcation region is maintained constant, i.e., $\tilde{n}=m-n$. If one considers the YJ composed of an infinite nanotube heterostructure at which a finite tube is attached composed of an ordered sequence of $l$ carbon rings (control arm), we call it as $m \rightarrow n / \tilde{n}_{l}$. Actually one may think that the finite arm acts as an idealized CNT control of varied extension[11]. Other YJ configurations have been recently studied, showing the dependence of interference effects on the topological arrangement of the arms[5].

A single $\pi$-band tight-binding calculation is used to describe the electronic properties of the CNT structures (pure carbon nanotubes, YJs and YJRs). Effects such as $\sigma-\pi$ hybridisation are not included in this one-band scheme. Rather then describing the system in the k-space, we treat the CNT-structures entirely in a real space picture. We follow the Green function formalism and adopt real-space renormalization group techniques $[9,12,13]$. The local density of states (LDOS) per electron is calculated directly from the relation $\rho_{i}(\omega)=-1 / \pi \operatorname{Tr}\left(\operatorname{Ima}\left[\tilde{G}_{i i}(\omega)\right]\right)$ where the dressed Green functions are obtained by solving a set of coupled matricial Dyson equations given by

$$
\tilde{G}_{i j}(\omega)=\tilde{g}_{i}(\omega) \delta_{i, j}+\tilde{g}_{i}(\omega) \sum_{l \neq i} V_{i l} \tilde{G}_{l j}(\omega)
$$

with $\tilde{g}_{i}$ denoting a matricial renormalized Green function corresponding to a single CNT ring, written in terms of the undressed $g_{i}(\omega)=1 /\left(\omega-\epsilon_{o}\right)$. The microscopic atomic details of the topological defect (heptagons) are taken into account in the theoretical description through hopping energy matrices $V_{i l}$, connecting the neighboring atomic layers (rings).

Results for the LDOS of an YJ $14 \rightarrow n / \tilde{n}$ with three semi-infinite CNTs are shown in Fig. 2, considering different combinations of arm sizes. A mean calculation is performed through all the atomic sites of a single ring, positioned two ring- layered from the defect. One should notice that semiconducting combinations of tubes, such as the $14 \rightarrow 7 / 7$ and $14 \rightarrow 4 / 10$ generate localized states within the energy gap. Combinations involving at least one metal- lic tube arm, such as $14 \rightarrow 6 / 8$ and $14 \rightarrow 5 / 9$, leads to the formation of a continuous band population within the gap. Due to the peculiar electronic properties of carbon nanotubes it is then possible to modulate the electronic response by selectively proposing proper Y-junctions with particular topology.

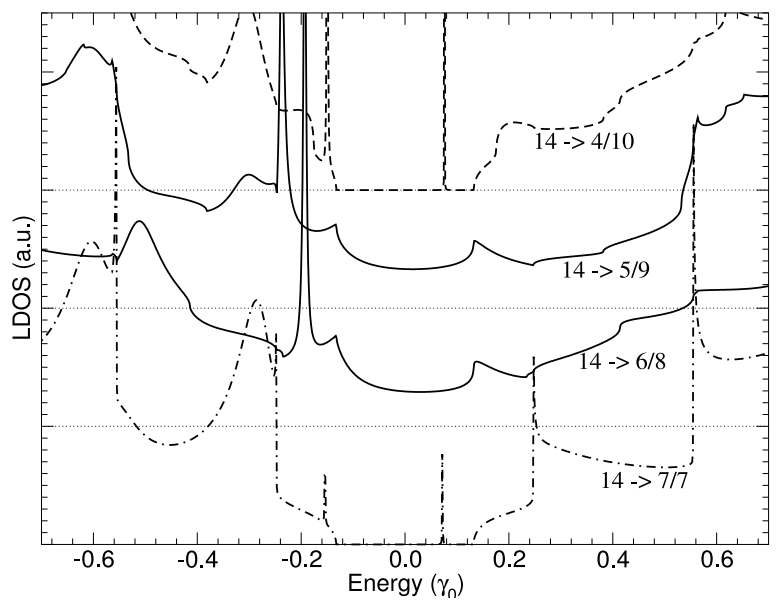

Figure 2. Local density of states near $E_{F}=0$ of symmetric and asymmetric YJs with big leg $(14,0)$. Curves are shifted for better viewing. When one of the legs is metallic, the LDOS near $E_{F}=0$ is non-zero.

The conductance may be written in terms of the dressed Green functions [13] by following the Landauer formalism[14] and the Kubo-Greenwood formula[15]. At the Fermi energy and for zero temperature one has

$$
\Gamma(\omega)=-\frac{2 e^{2}}{h} 2 T r \sum_{m n l k} \vec{R}_{m n} V_{m n} G_{n l}^{I+}(\omega) \cdot \vec{R}_{l k} V_{l k} G_{k m}^{I+}(\omega)
$$

in which $\vec{R}_{m n}=\vec{R}_{m}-\vec{R}_{n}, \vec{R}$ being the atomic site position, $G^{I+}$ means the imaginary part of the advanced Green function and the factor 2 is concerned to spin degeneracy. The present conductance calculation corresponds to the linear response of the total current density flowing through one tube into the other two. One should take care in this type of calculation about the role played by the imaginary part added to the energy $\omega+i \eta$. A finite $\eta$ defines an uncertainty in the energy, which is inversely proportional to an electronic transient-time. By performing calculations with $\eta \simeq 10^{-3}, 10^{-4}$ and considering the control arm finite, one gets spurious results for the conductance. In that sense, it is very important to take correctly the limit of $\eta \rightarrow 0$ in order to determine the localization properties of the electronic wave function.

Results for the conductance of a metallic YJ of type $12 \rightarrow 6 / 6_{L}$ for different values of $L$ (control tube length) are shown in Fig. 3. In the depicted energy range, where the conductance of a pure metallic CNT equals two units of a conductance quantum $2 e^{2} / h$ (two electronic channels), one may clearly observe a set of oscillations in the conductance as a function of the energy, associated probably with the presence of normal modes such as those of a resonant 
cavity, which number depends on its sizes. It is worth noticing that for sufficiently long control tubes (see the example of 100 control rings) a small variation in the number of carriers causes very large percentile changes of the system's conductance. Otherwise, an interesting result is the pinning of the conductance value at the Fermi energy for all YJs of finite control tube considered (when correctly taken the limit $\eta \rightarrow 0$ ). One clearly see that by varying the tube length one may modulate the conductance respectively (without getting zero-conductance), which rises the possibility of using the $\mathrm{YJ}$ as an idealized three-point transistor.

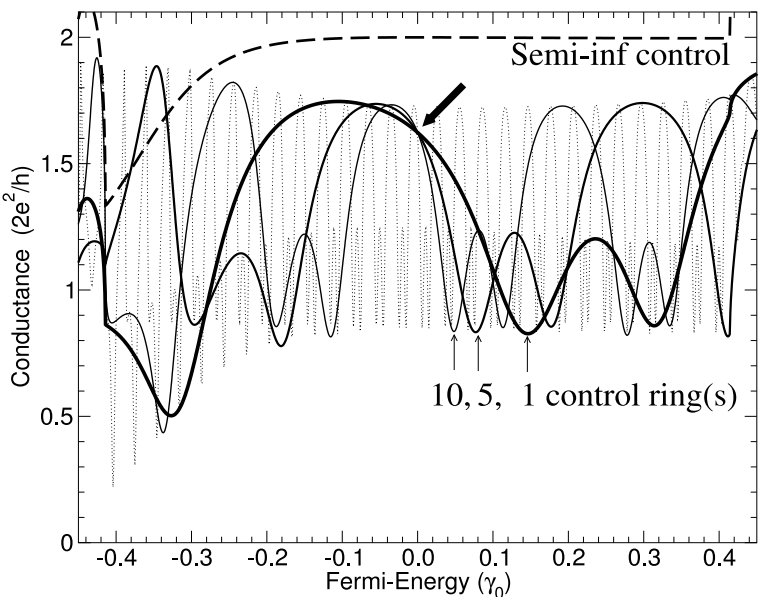

Fig. 3. Conductance vs. Fermi-energy of a symmetric $12 \rightarrow 6 / 6$ YJ with 1, 5, 10 and 100 (dotted line) rings in the control leg. Energy window is restricted to energies where $(12,0)$ has two conductance channels. Note the pinning of the conductance value at $E_{F}=0$, marked with an arrow.

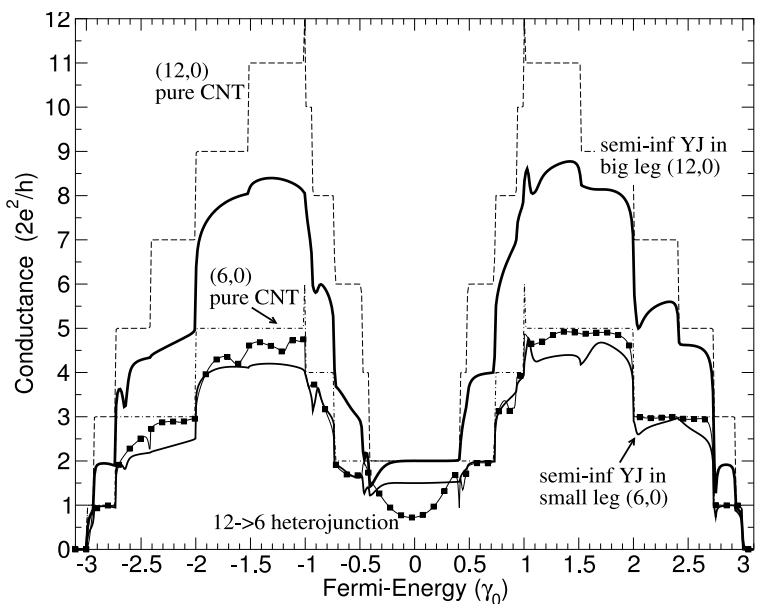

Figure 4. Conductance of symmetric a $12 \rightarrow 6 / 6 \mathrm{YJ}$ with three semi-infinite legs in comparison with a $12 \rightarrow 6$ heterojunction. The conductance channels of a pure $(12,0)$ and $(6,0)$ are shown with dashed lines.

For a single heterojunction of type $(12,0) \rightarrow(6,0)$, the greatest reduction of the conductance occurs at the center of the electronic band[9], i.e. at $E_{F}=0$ as shown in Fig. 4.
However, for the $12 \rightarrow 6 / 6 \mathrm{YJ}$ with three semi-infinite legs, the minimum occurs at the transition of a conductance channel of a pure metallic $(12,0) \mathrm{CNT}$. The conductance of YJs with finite control arm is, like in the case of the heterojunction, limited by the conductance channels of the smaller arm, i.e. six channels in the chosen $12 \rightarrow 6 / 6_{5}$ YJ. Otherwise, in the case of the semi-infinite YJ the conductance in each of the three semi-infinite legs is restricted by the dimension of their self size: the big $(12,0)$ arm has maximal twelve channels whereas both the two small $(6,0)$ arms have maximal six. Due to impurity scattering at the defect region, the conductance as a whole is reduced. One may also notice that due to quantum interference phenomena, the sum of the conductance of the small legs does not equal the conductance of the big one (contribution of mixed products of the electronic wave functions of each arm). As expected by the finite value of $\eta$ in the calculus, evaluating the semi-infinite limit one may find all the intermediate values on the way to the twelve-channel case. Furthermore, the conductance in the finite leg becomes non-zero for big control lengths, demonstrating the effect of the electronic transient time.

\section{Y-Junction Rings}

As discussed before, a ring-like nanotube structure may be idealized by joining two Y-Junctions presenting finite arms. Considering two $m \rightarrow n_{L 1 / 2} / \tilde{n}_{L 2 / 2}$ YJs, one may get a YJunction-Ring with upper and down arms composed of $L_{1}$ and $L_{2}$ rings, respectively. The so-built $m \rightarrow n_{L 1} / \tilde{n}_{L 2} \rightarrow$ $m$ YJR has two attached semi-infinite arms $(\mathrm{m}, 0)$ acting as a lead contact, where again $n+\tilde{n}=m$.

The easiest way to obtain the annular structure is by calculating renormalized non-diagonal Green function, obeying the recurrence relation

$$
\begin{aligned}
\tilde{G}_{i j} & =\tilde{G}_{i} V_{i, i-1} \tilde{G}_{i-1} \ldots \tilde{G}_{j+1} V_{j+1, j} \tilde{G}_{j}, \\
\text { with } \quad \tilde{G}_{i} & =\left(1-\tilde{g}_{i} V_{i, i-1} \tilde{g}_{i-1} V_{i-1, i}\right)^{-1} \tilde{g}_{i}
\end{aligned}
$$

for $i>j$ and assuming $\tilde{G}_{j}=\tilde{g}_{j}$ in the initial iterative step. We investigate here the possibility of generating quantum interference phenomena through the annular systems and study its correlation with the arm sizes composing the YJRs.

The dependence of the conductance of a (metallic) $12 \rightarrow$ $6_{L} / 6_{L} \rightarrow 12 \mathrm{YJR}$ in the energy window, where a pure $(12,0)$ tube has two ballistic channels, is shown in Fig. 5. This YJR is called asymmetric in the length of upper and lower braces between the two semi-infinite leads but symmetric in terms of the brace-tube size. The results for the LDOS of the corresponding system are also shown, illustrating the oscillatory behavior and correlation of both physical properties. Rather than the case of a single YJ, the conductance can be totally destroyed at particular energy values for certain arm configurations. Again, through barely geometrical configuration settings the YJR may be absolutely conducting or isolating, leading to a perfect rectification transistor. Symmetric YJRs exhibit completely different features: the conductance shows only smooth oscillations and the LDOS remains mainly constant at the central non-zero 
plateau of a pure $(12,0)$ tube, with isolated $\delta$-functions, indicating localized states. One may understand such features setting up a direct correspondence between these intricate carbon system and their one-dimensional counterpart, composed of an atomic loop connected to two metallic atomic leads, shown in the inset of the figure.

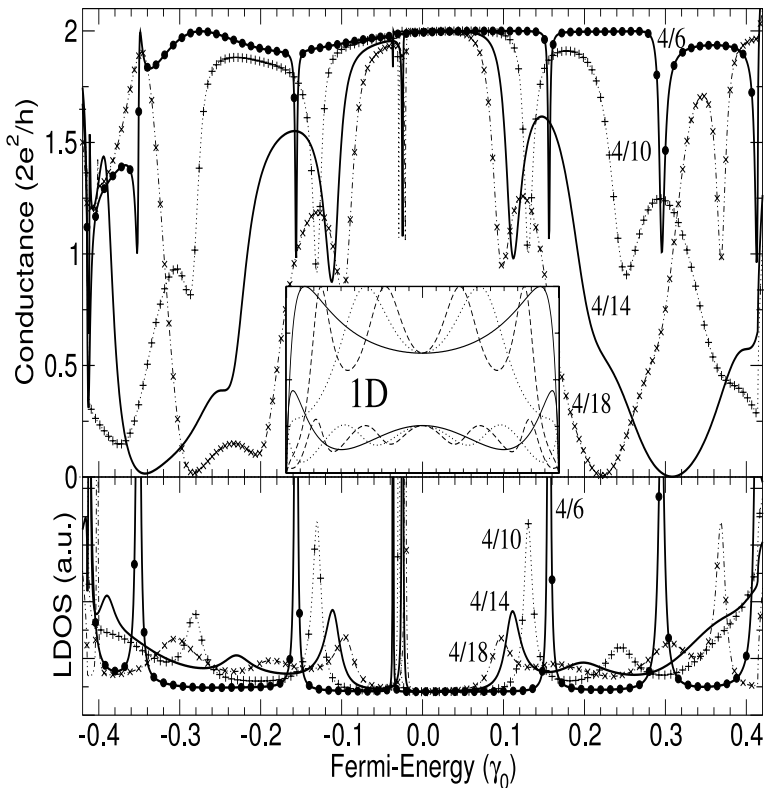

Figure 5. Conductance (top view) and LDOS (bottom view) of an asymmetric $12 \rightarrow 6_{4} / 6_{L} \rightarrow 12$ for different values of lower brace-length $(\mathrm{L}=6,10,14,18)$. Inset: Results for a one-dimensional symmetric counterpart, made of one atomic loop with 2 (straight), 4 (dotted) and 6 (dashed) atoms in each brace, connected to two metallic leads.

The LDOS of the 1D ring-system exhibits a sequence of peaks, positioned at energy values quite close to the discrete states composing the energy spectra of a single ring - which for the carbon YJR corresponds to a CNT-torus as previously studied[16]. Moreover, just as it happens in the $1 \mathrm{D}$ system and in a single $\mathrm{YJ}$, there is a perfect pinning of the LDOS at the Fermi level $\left(E_{F}=0\right)$ for all lengths of ring braces, provided one considers only even values and symmetrical brace-tube size.

As YJRs composed of semiconducting tubes do not allow conductance channels near the Fermi level one should suggest an extra junction formation to overcome this situation. Fig. 6 shows the conductance curves of the semi-conducting YJRs for some brace-lengths, this time connected to metallic leads through $(14,0) \rightarrow(12,0)$ heterojunctions[9]. As expected, the conductance and LDOS have finite values and are not zero as in the nonconnected case (shown as the dashed curve). Quite sharp peaks are obtained in energy range corresponding to the low band followed by smooth oscillation in the conductance and LDOS results. Here, the brace distributions (size and type) do not drive the main responses; actually it is the metallic $(12,0)$ lead which acts as the driving force for all configurations. Similar conductance and LDOS oscillatory behavior, with broken electron-hole symmetry, are also found for YJR presenting asymmetric brace-tube sizes, such as $12 \rightarrow 5_{L 1} / 7_{L 2} \rightarrow 12$

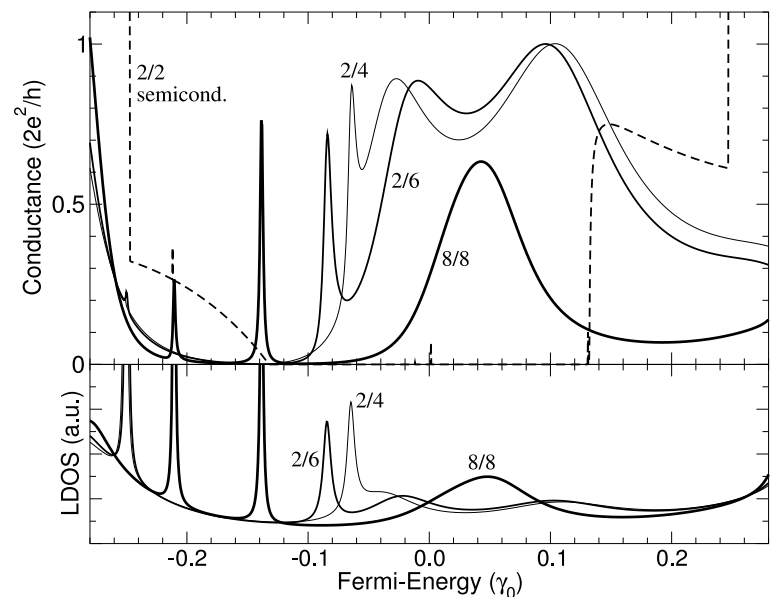

Figure 6. Conductance of a $14 \rightarrow 7 / 7 \rightarrow 14$ YJR connected to metallic lead through $(14,0) \rightarrow(12,0)$ heterojunctions in the energy range, where a pure $(14,0)$ has zero- conductance. A pure $14 \rightarrow 7 / 7 \rightarrow 14$ YJR with zero-conductance near the central gap is shown with dashed lines.

\section{Conclusions}

We have studied the electronic LDOS and conductance near the Fermi level of single Y-junctions and two-joined YJs, forming a Y-junction ring and compared with onedimensional counterpart examples. In all situations we observed oscillatory behavior depending merely on geometrical configuration. In the case of the Y-junction, the control of one of the length-brace can lead to perfect switching behavior. In the case of the YJR, the position of the leads depending on the annular sites can give rise to complete destructive or constructive quantum interference. Since transport phenomena through varied nanotube structures are believed to be promising for technological applications, like a three-point nanotransistor or nanoscale molecular circuit including a carbon YJR, the understanding of their electronic properties is essential for further development in this field.

\section{Acknowledgments}

We would like to thank Drs. M. F. dos Santos for useful discussions. This work was partially supported by the Brazilian Agencies CNPq,CAPES, and FAPERJ.

\section{References}

[1] C. Papadopoulos, A. Rakitin, J. Li, A.S. Vedeneev and J.M. Xu, Phys. Rev. Lett. 85, 3476 (2000).

[2] M. Terrones, F. Banhart, N. Grobert, J.-C. Charlier, H. Terrones and P.M. Ajayan, Phys. Rev. Lett. 89, 75505 (2002).

[3] M. Terrones, H. Terrones, F. Banhart, J.-C. Charlier, and P.M. Ajayan, Science 288, 1226 (2000). 
[4] L.P. Biró, R. Ehlich, Z. Osváth, A. Koós, Z.E. Horváth, J. Gyulai, J.B. Nagy; Diamond and Related Materials 11, 1081 (2002).

[5] A. Andriotis, M. Menon, D. Srivastava and L. Chernozatonskii; Phys. Rev. B 65, 165416 (2002).

[6] H. Watanabe, C. Manabe, T. Shigematsu and M. Shimizu, Appl. Phys. Lett. 78, 2928 (2002).

[7] G. Cuniberti, J. Yi and M. Porto, Appl. Phys. Lett. 81, 850 (2002).

[8] L. A. Chernozatonskii, Phys. Lett. A 172, 173 (1992).

[9] M. S. Ferreira, T. G. Dargam, R. B. Muniz and A. Latgé, Phys. Rev B 62, 16040 (2000).

[10] V. H. Crespi, Phys. REv. B 58,12671 (1998).
[11] G. Treboux, P. Lapstun, Z. Wu, and K. Silverbrook, J. Phys. Chem. B 103, 8671 (1999).

[12] M. S. Ferreira, T. G. Dargam, R.B. Muniz and A. Latgé, Phys. Rev B 63, 245111 (2001)

[13] A. Latgé, D. C. Marcucci e M. V. Tovar Costa, Physica E 13, 1264 (2002); C. G. Rocha, T. G. Dargam, and A. Latgé, Phys Rev. B 65 165431, (2002).

[14] J. Mathon, M. Villeret, and H. Itoh, Phys. Rev. B 52, R6983 (1995).

[15] R. Kubo, J. Phys. Soc. Jpn, 12, 570 (1957).

[16] A. Latgé, C. G. Rocha, L. A. Lavenére, P. Orellana, M. Pacheco, and Z. Barticevic, Phys. Rev. B 67, 155413 (2003). 